\title{
Long non-coding RNAs regulate effects of $\beta$-crystallin B2 on mouse ovary development
}

\author{
QIAN GAO*, HANXIAO REN*, MINGKUN CHEN, ZIGUANG NIU, \\ HAIBO TAO, YIN JIA, JIANRONG ZHANG and WENJIE LI \\ Department of Laboratory Diagnosis, Changhai Hospital, The Second Military Medical University, \\ Shanghai 200433, P.R. China
}

Received September 2, 2015; Accepted September 6, 2016

DOI: $10.3892 / \mathrm{mmr} .2016 .5761$

\begin{abstract}
B2 (CRYBB2) knockout mice exhibit morphological and functional abnormalities in the ovary. Long non-coding RNAs (lncRNAs) regulate gene transcription and translation, and epigenetic modification of genomic DNA. The present study investigated the role of lncRNAs in mediating the effects of CRYBB2 in the regulation of ovary development in mice. In the current study, ovary tissues from wild-type (WT) and CRYBB2 knockout mice were subjected to IncRNA and mRNA microarray profiling. Gene Ontology (GO) and Kyoto Encyclopedia of Genes and Genomes (KEGG) analyses were performed to group the differentially expressed IncRNAs into regulated gene pathways and functions. The correlation matrix method was used to establish a network of lncRNA and mRNA co-expression. Quantitative reverse transcription-polymerase chain reaction (RT-qPCR) was used to verify expression of a number of these differentially expressed lncRNAs and mRNAs. There were 157 differentially expressed lncRNAs and 1,085 differentially expressed mRNAs between ovary tissues from WT and CRYBB2 knockout mice. The GO and KEGG analyses indicated that these differentially expressed lncRNAs and mRNAs were important in $\mathrm{Ca}^{2+}$ signaling and ligand and receptor interactions. The correlation matrix method established an IncRNA and mRNA co-expression network, consisting of $53 \mathrm{lncRNAs}$ and 45 mRNAs with 98 nodes and 75 connections. RT-qPCR confirmed downregulation of lncRNA A-30-P01019163 expression, which further downregulated its downstream gene purinergic receptor P2X, ligand-gated ion channel, 7 (P2rx7) expression
\end{abstract}

Correspondence to: Dr Wenjie Li, Department of Laboratory Diagnosis, Changhai Hospital, The Second Military Medical University, 168 Changhai Road, Shanghai 200433, P.R. China

E-mail: wenjieli@pku.org.cn

${ }^{*}$ Contributed equally

Key words: $\beta$-crystallin B2, long non-coding RNAs, ovary development, signal transduction in ovary tissues from $C R Y B B 2$ knockout mice. In conclusion, CRYBB2 regulates expression of different lncRNAs to influence ovary development. IncRNA A-30-P01019163 may affect ovarian cell cycle and proliferation by regulating P2rx7 expression in the ovary.

\section{Introduction}

$\beta$-crystallin B2 (CRYBB2) expression was initially reported in the eye lens where it functions to maintain lens transparency and refractive index. CRYBB2 deficiency was demonstrated to result in the generation of age-associated cataracts (1). However, a number of previous studies demonstrated that CRYBB2 is also expressed in the retina, brain, testis, and ovary (2-5). Our previous study observed that CRYBB2 was expressed in human and mouse ovaries, particularly in ovarian granulosa cells (6). CRYBB2 knockout mice exhibited morphological and functional abnormalities in the ovary, including reduced ovarian index (ratio of ovary weight to total body weight) with increased follicle atresia, reduced mature follicles and dysregulated estrous cycle (7). Our data from a previous study also indicated a high level of estrogen in the diestrus and metestrus, but a low level of progesterone in the metestrus compared with wild-type (WT) mice (6). At the genetic level, expression of cell cycle and apoptosis-associated proteins, including B-cell lymphoma 2, cyclin-dependent kinase 4, and cyclin D2, were markedly lower in the $C R Y B B 2$ knockout mice compared with WT mice (6). These data suggest that $C R Y B B 2$ may be important in ovary development, however, the underlying molecular mechanism by which $C R Y B B 2$ regulates ovarian development remains to be elucidated.

To identify and assess the role of CRYBB2 in ovary development, differentially expressed long non-coding RNAs (lncRNAs) were profiled in CRYBB2 knockout mice. IncRNAs are a class of non-coding RNAs with nucleotides $>200 \mathrm{bp}$ and transcribed by RNA polymerase II. Functionally, lncRNAs may regulate gene transcription, protein translation, and epigenetic modification of genomic DNA. Altered expression and regulation of lncRNAs has been associated with human diseases and aging (8-12). For example, previous studies have suggested that overexpression of IncRNA HOX transcript antisense RNA associated with the recurrence of hepatocellular 
carcinoma, poor prognosis in colorectal cancer, and malignant behaviors of gastrointestinal stromal tumors (13-15). lncRNAs modulate cell functions by regulating expression of targeted downstream genes (16), which may in turn affect embryo development (17), inactivate the $\mathrm{X}$ chromosome, and regulate genomic imprinting (18). Thus, the current study assessed whether these lncRNAs mediate the functions of CRYBB2 in ovary development and investigated the underlying mechanisms. Microarray profiling between ovarian tissues from $C R Y B B 2$ knockout and WT mice was conducted and bioinformatic analysis of differentially expressed lncRNAs and mRNAs was performed. A number of these differentially expressed lncRNAs and mRNAs were verified using quantitative reverse transcription-polymerase chain reaction (RT-qPCR).

\section{Materials and methods}

Animals. Male and female C57BL/6 mice were obtained from the Experimental Animal Center of the Second Military Medical University (Shanghai, China). CRYBB2 knockout mice were generated by the Ingenious Targeting Laboratory (Ronkonkoma, NY, USA), as described previously (19). All mice were maintained on a $12 \mathrm{~h}$ light/dark cycle with a temperature of $21 \pm 1^{\circ} \mathrm{C}$ and humidity of $50 \sim 70 \%$ in a pathogen-free facility with access to food and water ad libitum. Bedding material and a plastic house or tube was placed in the cage for environmental enrichment. Daily examinations were performed on all animals throughout the experimental period. Humane euthanasia of mice was performed under isoflurane anesthesia using intracardiac injection of pentobarbitone $(150 \mathrm{mg} / \mathrm{kg})$. The present study was conducted in accordance with institutional guidelines and approved by the Animal Care and Use Committee of Changhai Hospital (Shanghai, China).

A total of three 8-9-week-old (weight, 18.0 $2.0 \mathrm{~g}$ ) female $C R Y B B 2$ knockout mice and three age-matched WT female mice were obtained. Ovary tissues were collected from mice following a 10-day experimental period.

RNA isolation, cDNA synthesis and labeling, and hybridization. Ovarian tissues were homogenized on ice and total cellular RNA was isolated using TRIzol reagent (Invitrogen; Thermo Fisher Scientific, Inc., Waltham, MA, USA) according to the manufacturer's protocol and then quantified using NanoDrop ND-1000 (Thermo Fisher Scientific, Inc., Wilmington, DE, USA) and agarose gel electrophoresis. RNA samples were further purified using an RNeasy Mini kit (Qiagen, Inc., Valencia, CA, USA) and reverse transcribed into cDNA with fluorescent labeling for microarray hybridization using using the AffinityScript QPCR cDNA Synthesis kit (Agilent Technologies, Inc., Santa Clara, CA, USA). These labeled cDNA probes were then hybridized to Agilent mouse expression profiling $(8 \times 60 \mathrm{~K})$ microarray using the Gene Expression Hybridization kit (Agilent Technologies, Inc.) according to the manufacturer's protocol. The arrays were scanned into a file and analyzed using Feature Extraction software, version v10.7.3.1 (Agilent Technologies, Inc.). The arrays were scanned at $5 \mu \mathrm{m} /$ pixel resolution using an Axon GenePix 4000B scanner
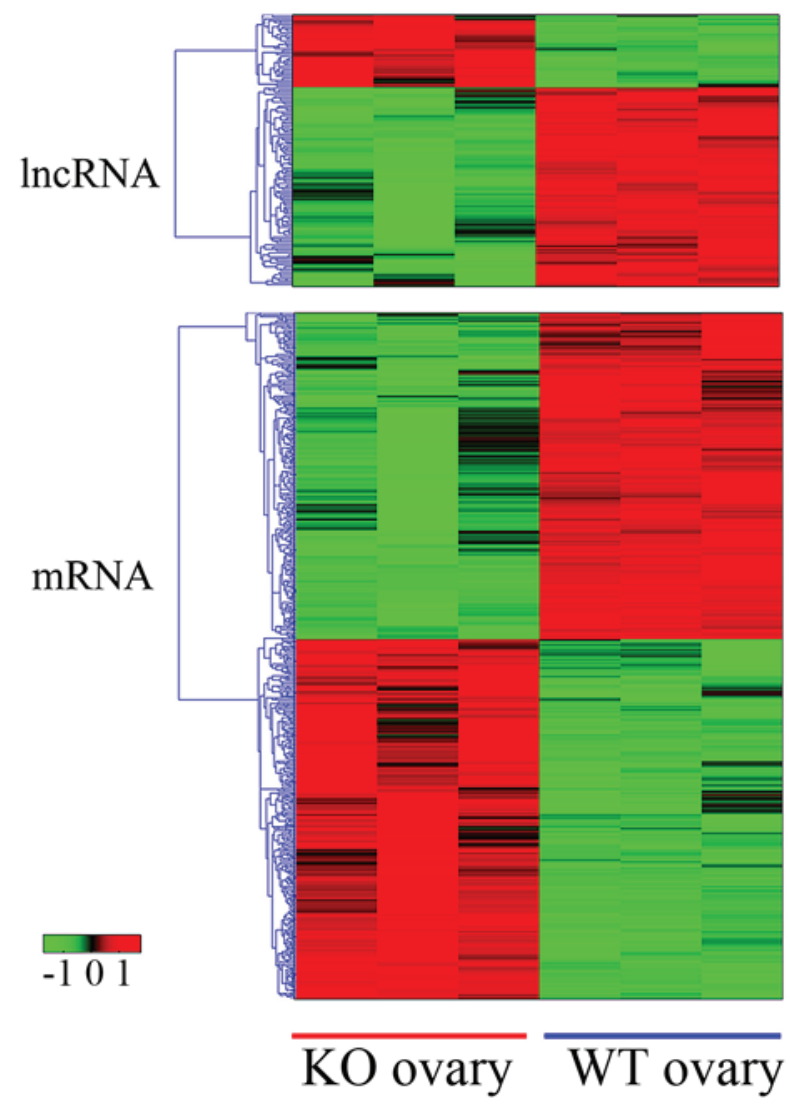

Figure 1. Cluster analysis of differentially expressed lncRNAs and mRNAs in ovary tissues from WT and $\beta$-crystallin B2 knockout mice. The horizontal axis represents the sample group and the vertical axis represents the differentially expressed mRNAs or lncRNAs. Upregulated genes are red and downregulated genes are green. Included are 157 lncRNAs (42 downregulated vs. 115 upregulated) and 1,085 mRNAs (570 downregulated vs. 515 upregulated) KO, knockout; WT, wild-type; lncRNA, long non-coding RNA.

(Molecular Devices, LLC, Sunnyvale, CA, USA) piloted by GenePix Pro 6.0 software (Molecular Devices, LLC) and then imported into NimbleScan software (version 2.5; Roche NimbleGen, Inc., Madison, WI, USA) for grid alignment and expression data analysis. Expression data were normalized using quantile normalization and the Robust Multichip Average algorithm included in the NimbleScan software. The probe level files and mRNA level files were generated following normalization. All mRNA level files were imported into Agilent GeneSpringGX software (version 11.0; Agilent Technologies, Inc.) for further analysis. Differentially expressed lncRNAs and mRNAs were identified via fold change filtering.

Functional analysis of microarray data. Gene Ontology (GO; www.geneontology.org) was performed to group differentially expressed lncRNAs and their targeted genes into biological processes, cellular components, and molecular features of the biological functions. Kyoto Encyclopedia of Genes and Genomes (KEGG; www.genome.jp/kegg) analysis was performed to identify roles of the target genes and group them into gene pathways. The correlation matrix method was used to establish a diagram of IncRNA and mRNA co-expression regulatory networks. Prior to functional analyses, the 
Table I. Differentially expressed long non-coding RNAs in ovary tissues from $\beta$-crystallin B2 knockout mice.

\begin{tabular}{|c|c|c|c|}
\hline Probe name & P-value & FC (abs) & Regulation \\
\hline A_30_P01032506 & 0.001900622 & 2.244835 & Down \\
\hline A_30_P01029732 & 0.026376737 & 2.3413253 & Down \\
\hline A_30_P01020038 & 0.020382054 & 2.3550973 & Down \\
\hline A_30_P01019163 & 0.008867065 & 2.3823233 & Down \\
\hline A_30_P01017808 & 0.003141625 & 2.3902857 & Down \\
\hline A_30_P01028465 & 0.026580842 & 2.3909771 & Down \\
\hline A_30_P01024278 & 0.010205811 & 2.4727218 & Down \\
\hline A_30_P01031572 & 0.036648612 & 2.5956807 & Down \\
\hline A_30_P01027590 & 0.034441914 & 2.6343288 & Down \\
\hline A_30_P01022135 & 0.04770927 & 3.120179 & Down \\
\hline A_30_P01031631 & 0.001265911 & 3.135137 & Down \\
\hline A_30_P01032372 & 0.009654216 & 3.4001627 & Down \\
\hline A_30_P01032133 & 0.012271924 & 3.434039 & Down \\
\hline A_30_P01024342 & $2.97 \times 10^{-4}$ & 3.7296832 & Down \\
\hline A_30_P01024108 & 0.004697592 & 3.8198798 & Down \\
\hline A_30_P01022445 & $2.66 \times 10^{-5}$ & 3.8623033 & Down \\
\hline A_30_P01022656 & $2.08 \times 10^{-4}$ & 4.069888 & Down \\
\hline A_30_P01023636 & 0.02164327 & 4.4273615 & Down \\
\hline A_30_P01022538 & $9.77 \times 10^{-4}$ & 5.492365 & Down \\
\hline A_30_P01033546 & 0.004913332 & 5.9940124 & Down \\
\hline A_30_P01019023 & 0.024659809 & 3.7894382 & Up \\
\hline A_30_P01024108 & 0.004697592 & 3.8198798 & Up \\
\hline A_30_P01017880 & 0.034943227 & 3.8256395 & Up \\
\hline A_30_P01022445 & $2.66 \times 10^{-5}$ & 3.8623033 & Up \\
\hline A_30_P01026472 & 0.022304738 & 3.8728366 & Up \\
\hline A_30_P01030900 & 0.023211088 & 3.9997435 & Up \\
\hline A_30_P01022656 & $2.08 \times 10^{-4}$ & 4.069888 & Up \\
\hline A_30_P01018745 & $1.84 \times 10^{-5}$ & 4.266393 & Up \\
\hline A_30_P01033353 & 0.03583063 & 4.398616 & Up \\
\hline A_30_P01021636 & 0.01934708 & 4.4207296 & Up \\
\hline A_30_P01023636 & 0.02164327 & 4.4273615 & Up \\
\hline A_30_P01022538 & $9.77 \times 10^{-4}$ & 5.492365 & Up \\
\hline A_30_P01019825 & 0.006051722 & 5.5902176 & Up \\
\hline A_30_P01033546 & 0.004913332 & 5.9940124 & Up \\
\hline A_30_P01024788 & $1.11 \times 10^{-5}$ & 7.118162 & Up \\
\hline A_30_P01024270 & $6.38 \times 10^{-5}$ & 8.226261 & Up \\
\hline A_30_P01027087 & 0.003292078 & 8.981714 & Up \\
\hline A_30_P01031162 & 0.011685452 & 9.579193 & Up \\
\hline A_30_P01021698 & $1.31 \times 10^{-5}$ & 10.52238 & Up \\
\hline A_30_P01020936 & $4.52 \times 10^{-4}$ & 22.788074 & Up \\
\hline
\end{tabular}

FC, fold change.

predicted potential IncRNA targets were integrated with the differentially expressed mRNAs using a cut-off value of $\geq 2.0$ fold change or $\mathrm{P}<0.05$.

$R T$ - $q P C R$. Total RNA was isolated from granulosa cells from the ovaries of WT and CRYBB2 knockout mice using TRIzol reagent and reverse transcribed into cDNA using the
PrimeScript RT Reagent Kit (Takara Biotechnology, Co., Ltd., Dalian, China) according to the manufacturer's protocols. These cDNA samples were then subjected to qPCR analysis using a Applied Biosystems 7300 Real-Time PCR system (Applied Biosystems; Thermo Fisher Scientific, Inc.) with the SYBR Green PCR Master Mix kit (Takara Biotechnology, Co., Ltd.). qPCR amplification was conducted at $95^{\circ} \mathrm{C}$ for 
Table II. Differentially expressed mRNAs in ovary tissues from $\beta$-crystallin B2 knockout mice.

\begin{tabular}{|c|c|c|c|}
\hline Gene & P-value & FC (abs) & Regulation \\
\hline Prkar2b & 0.040216 & 2.1857524 & Down \\
\hline Lrp11 & 0.006975 & 2.9040618 & Down \\
\hline $\mathrm{P} 2 \mathrm{rx} 7$ & $4.11 \times 10^{-4}$ & 4.6213336 & Down \\
\hline Calml3 & 0.011701 & 5.126569 & Down \\
\hline Dclre1c & $3.39 \times 10^{-4}$ & 5.1453366 & Down \\
\hline Lpcat2 & 0.002695 & 5.828531 & Down \\
\hline Stk32a & 0.001242 & 5.833555 & Down \\
\hline Cyp19a1 & 0.034041 & 6.1799 & Down \\
\hline Megf10 & 0.045621 & 6.1827664 & Down \\
\hline Plcxd1 & $1.04 \times 10^{-4}$ & 6.82413 & Down \\
\hline Gm5103 & 0.005622 & 8.389297 & Down \\
\hline Fermt 1 & 0.001363 & 8.656997 & Down \\
\hline Mlxip & $3.10 \times 10^{-4}$ & 8.746344 & Down \\
\hline Slc6a2 & 0.046581 & 8.750824 & Down \\
\hline Gm7969 & 0.012795 & 9.970471 & Down \\
\hline Ces $2 a$ & 0.028129 & 10.472376 & Down \\
\hline Dclre1c & $1.23 \times 10^{-4}$ & 10.645219 & Down \\
\hline Ostn & $9.07 \times 10^{-6}$ & 11.019576 & Down \\
\hline Jph4 & 0.001674 & 12.722166 & Down \\
\hline Onecut2 & 0.048542 & 13.894124 & Down \\
\hline Ces $2 a$ & 0.028128909 & 10.472376 & Up \\
\hline Dclre1c & $1.23 \times 10^{-4}$ & 10.645219 & Up \\
\hline Jph4 & 0.001673621 & 12.722166 & Up \\
\hline Onecut2 & 0.04854157 & 13.894124 & Up \\
\hline Itln 1 & $5.97 \times 10^{-5}$ & 14.339393 & Up \\
\hline Arsk & $7.13 \times 10^{-5}$ & 16.06716 & Up \\
\hline Sfrp4 & 0.023781205 & 17.065975 & Up \\
\hline Plekhg4 & 0.047409292 & 17.067673 & Up \\
\hline Nuf2 & $6.05 \times 10^{-5}$ & 17.872303 & Up \\
\hline Adh7 & 0.0064019 & 18.950857 & Up \\
\hline Itln 1 & $4.63 \times 10^{-5}$ & 19.05549 & Up \\
\hline Ptgfr & 0.032550577 & 20.059986 & Up \\
\hline Pou6f1 & $1.71 \times 10^{-4}$ & 23.766891 & Up \\
\hline Saa2 & 0.023944996 & 25.063555 & Up \\
\hline Wnt10b & 0.02046059 & 27.648937 & Up \\
\hline Ifi202b & 0.001051159 & 28.72067 & Up \\
\hline Dcpp1 & 0.014649089 & 40.37458 & Up \\
\hline $\mathrm{Cd} 51$ & $4.12 \times 10^{-4}$ & 42.74245 & Up \\
\hline Dcpp2 & 0.012848383 & 45.13117 & Up \\
\hline
\end{tabular}

FC, fold change.

$2 \mathrm{~min}$, followed by 40 cycles of $95^{\circ} \mathrm{C}$ for $15 \mathrm{sec}$, and $60^{\circ} \mathrm{C}$ for $30 \mathrm{sec}$. The relative expression of each target gene compared to $\beta$-actin was calculated using the $2^{-\Delta \Delta C q}$ method (20). Specific primers used were as follows: Forward, 5'-AGCCATGTACGT AGCCATCC-3' and reverse, 5'-CTCTCAGCTGTGGTGGTG AA-3' for $\beta$-actin; forward, 5'-CGAGTTGGTGCCAGTGTG GA-3' and reverse, 5'-CCTGCTGTTGGTGGCCTCTT-3' for P2rx7; and forward, 5'-TCCACTCAGGAAGAGCTG
GT-3' and reverse, 5'-TAGCACCCTCGGGATATCTG-3' for IncRNAA-30-P01019163.

Statistical analysis. All data were presented as the mean \pm standard deviation. The data were Log2-transformed and median centered by genes using Cluster software, version 3.0 (http://bonsai.hgc.jp/ mdehoon/software/cluster/software.htm). Statistical analyses were performed using SPSS 17.0 (SPSS, 
Table III. Gene ontology analysis of differentially expressed genes.

\begin{tabular}{|c|c|c|}
\hline Term & Number of genes $(\%)$ & P-value \\
\hline \multicolumn{3}{|l|}{ Biological process } \\
\hline Cell cycle and proliferation & $3(4)$ & 0.479979 \\
\hline Stress response & $4(6)$ & 0.205102 \\
\hline Transport & $5(7)$ & 0.563981 \\
\hline Developmental processes & $6(9)$ & 0.459856 \\
\hline RNA metabolism & $7(10)$ & 0.315857 \\
\hline Other metabolic processes & $12(17)$ & 0.007676 \\
\hline Cell organization and biogenesis & $6(9)$ & 0.193590 \\
\hline Cell-cell signaling & $1(1)$ & 0.502761 \\
\hline Signal transduction & $11(16)$ & 0.120754 \\
\hline Protein metabolism & $6(9)$ & 0.502818 \\
\hline Death & $1(1)$ & 0.827556 \\
\hline Other biological processes & $8(11)$ & 0.990425 \\
\hline \multicolumn{3}{|l|}{ Cellular component } \\
\hline Cytosol & $1(2)$ & 0.505969 \\
\hline Mitochondrion & $1(2)$ & 0.862146 \\
\hline Endoplasmic reticulum/golgi & $3(5)$ & 0.460760 \\
\hline Other cytoplasmic organelle & $1(2)$ & 0.604395 \\
\hline Nucleus & $10(18)$ & 0.306385 \\
\hline Plasma membrane & $6(1)$ & 0.438812 \\
\hline Other membranes & $14(26)$ & 0.661329 \\
\hline Translational apparatus & $1(2)$ & 0.330956 \\
\hline Non-structural extracellular & $2(4)$ & 0.830334 \\
\hline Other cellular component & $15(28)$ & 0.354726 \\
\hline \multicolumn{3}{|l|}{ Molecular function } \\
\hline Enzyme regulator activity & $3(5)$ & 0.141417 \\
\hline Transcription regulatory activity & $4(7)$ & 0.174629 \\
\hline Transporter activity & $2(4)$ & 0.623057 \\
\hline Signal transduction activity & $11(20)$ & 0.082302 \\
\hline Nucleic acid binding activity & $7(13)$ & 0.252320 \\
\hline Kinase activity & $2(4)$ & 0.503159 \\
\hline Other molecular function & $26(47)$ & 0.535279 \\
\hline
\end{tabular}

Inc., Chicago, IL, USA). The data were statistically analyzed using an unpaired Student's t-test or by one-way analysis of variance followed by Tukey's test. $\mathrm{P}<0.05$ was considered to indicate a statistically significant difference.

\section{Results}

Microarray profiling of differentially expressed lncRNAs and $m R N A$ in ovary tissues from wild type and CRYBB2 knockout mice. Microarray data on differential lncRNA and mRNA expression levels were analyzed using Cluster software, version 3.0 with the cut-off values set as $\geq 2$-fold difference or $\mathrm{P}<0.05$. The data indicated 157 differentially expressed lncRNAs (42 downregulated vs. 115 upregulated) and 1,085 differentially expressed mRNAs (570 downregulated vs. 515 upregulated; Fig. 1). The genes were selected according to a software forecast associated with the processes of ovarian development, and the lncRNAs associated with these genes were selected (Tables I and II).
Bioinformatic analysis of differentially expressed lncRNAs and $m R N A$ in ovary tissues from WT and CRYBB2 knockout mice. GO analysis was performed for functional annotation of differentially expressed lncRNAs and mRNAs and it was observed that they were predominantly involved in cell cycle regulation, cell proliferation, metabolism, and signal transduction (Table III). One particular gene, P2rx7, localized in the cytoplasm, functions to regulate cell cycle progression and proliferation, and the signal transduction process.

Subsequently, KEGG analysis was conducted to group differentially expressed lncRNAs and mRNA into gene pathways. The data from the present study indicated that the genes predominantly formed signaling pathways associated with the regulation of $\mathrm{Ca}^{2+}$ signaling, ligand and receptor interactions, and other cell signaling pathways (Table IV). For example, $\mathrm{P} 2 \mathrm{rx} 7$ was identified to be predominantly involved in $\mathrm{Ca}^{2+}$ signaling. 
Table IV. KEGG pathways analysis of differentially expressed genes.

\begin{tabular}{|c|c|c|c|c|c|}
\hline Term & Count & P-value & Genes & Up & Down \\
\hline Glutathione metabolism & 1 & 0.106032 & Gsta2 & 0 & 1 \\
\hline Metabolism of xenobiotics by cytochrome $\mathrm{P} 450$ & 1 & 0.143567 & Gsta2 & 0 & 1 \\
\hline Drug metabolism-cytochrome P450 & 1 & 0.147434 & Gsta2 & 0 & 1 \\
\hline Ribosome & 1 & 0.172182 & Rps3a & 0 & 1 \\
\hline PPAR signaling pathway & 1 & 0.143567 & Adipoq & 0 & 1 \\
\hline Calcium signaling pathway & 3 & 0.00614 & P2rx7, Ptger1, Ptafr & 3 & 0 \\
\hline Cytokine-cytokine receptor interaction & 1 & 0.45143 & Cxcl1 & 0 & 1 \\
\hline Chemokine signaling pathway & 1 & 0.353888 & Cxcl1 & 0 & 1 \\
\hline Neuroactive ligand-receptor interaction & 4 & 0.001745 & P2rx7, Ptger1, Ptafr, Vipr1 & 4 & 0 \\
\hline Cell cycle & 1 & 0.226819 & Hdac 1 & 0 & 1 \\
\hline Apoptosis & 1 & 0.179664 & Prkar2b & 1 & 0 \\
\hline Notch signaling pathway & 1 & 0.099971 & Hdac1 & 0 & 1 \\
\hline Insulin signaling pathway & 1 & 0.268146 & Prkar2b & 1 & 0 \\
\hline Adipocytokine signaling pathway & 1 & 0.139685 & Adipoq & 0 & 1 \\
\hline Type II diabetes mellitus & 1 & 0.099971 & Adipoq & 0 & 1 \\
\hline Huntington's disease & 1 & 0.334126 & Hdac1 & 0 & 1 \\
\hline Pathways in cancer & 1 & 0.530933 & Hdac1 & 0 & 1 \\
\hline Chronic myeloid leukemia & 1 & 0.155119 & Hdac1 & 0 & 1 \\
\hline
\end{tabular}

Building the IncRNA-mRNA regulatory network. The IncRNA target predictions were superimposed into the IncRNA-mRNA correlation network. As presented in Fig. 2, the network has a total of 98 nodes, 75 connections, 53 lncRNAs and 45 mRNAs. Expression of glutathione S-transferase $\alpha 2$ (Gsta2) and DEAD (Asp-Glu-Ala-Asp) box polypeptide $43(\mathrm{Ddx} 43)$ was regulated by a total of five lncRNAs, while expression of coiled-coil domain containing 68 (Ccdc68), olfactory receptor 464 (Olfr464) and low density lipoprotein receptor-related protein 11 (Lrp11) were regulated by four IncRNAs. In addition, expression levels of protein kinase, cAMP dependent regulatory, type II $\beta$ (Prkar2b), leucine-rich repeat-containing $G$ protein-coupled receptor 6 (Lgr6) and adiponectin, C1Q and collagen domain containing (Adipoq) were regulated by a total of three lncRNAs.

Validation of differentially expressed lncRNAs and mRNAs in ovary tissues of WT and CRYBB2 knockout mice. RT-qPCR analysis of different lncRNAs and mRNAs was performed and it was demonstrated that expression levels of 1ncRNA A-30-P01019163 and P2rx7 were significantly different between 10 cases of ovary tissues from WT and $C R Y B B 2$ knockout mice $(\mathrm{P}<0.05$; Fig. 3), whereas other IncRNAs and mRNAs did not indicate any difference between WT and $C R Y B B 2$ knockout mouse ovary tissues (data not shown).

\section{Discussion}

CRYBB2 knockout mice exhibit abnormal morphological and functional mouse ovaries. The current study investigated the underlying molecular mechanisms by which $C R Y B B 2$ affects expression of lncRNAs to alter mouse ovary structure and function. Certain aberrant lncRNA expression in the regulation of other genes was assessed. A total of 157 differentially expressed lncRNAs and 1,085 mRNAs were observed. The $\mathrm{GO}$ database analysis indicated that these altered gene expressions and were predominantly distributed in the biological process of metabolism, immune system, and signal transduction. The KEGG database pathway analysis suggested that the predominant signaling pathways were associated with $\mathrm{Ca}^{2+}$ signaling and ligand-receptor interaction.

Subsequent to establishing a correlation matrix lncRNA and mRNA co-expression network map, a total of 98 nodes, 75 connections, 53 lncRNAs, and 45 mRNAs were identified. Gsta2 and Ddx43 were regulated by five lncRNAs, Ccdc68, Olfr464 and Lrp11 by four lncRNAs, Prkar2b, Lgr6, and Adipoq by three lncRNAs. Gsta2 participates in cytochrome P450 metabolism and cytochrome P450 is important in the conversion of androgens to estrogens. P2rx7, Olfr464, Lrp11, Prkar2b, Lgr6, and Adipoq are involved in cellular signal transduction and P2rx7, Prkar2b, and Hdacl are involved in regulation of the cell cycle and cell proliferation, while Prkar2b, Hdac1, and Dock7 are important in cell development. IncRNA A-30-P01019163 and $\mathrm{P} 2 \mathrm{rx} 7$ were differentially expressed in ovarian tissues of $C R Y B B 2$ knockout mice.

Crybb2 not only functions to maintain lens transparency and refractive index, but also affects ovary development in mice. At the gene level, Crybb2 may mediate $\mathrm{Ca}^{2+}$ signal transduction $(21,22)$. The current study demonstrated that Crybb2 also regulates expression of lncRNAs in ovarian tissues, which may be a novel area of research on Crybb2-regulation of gene expression. Previous studies 

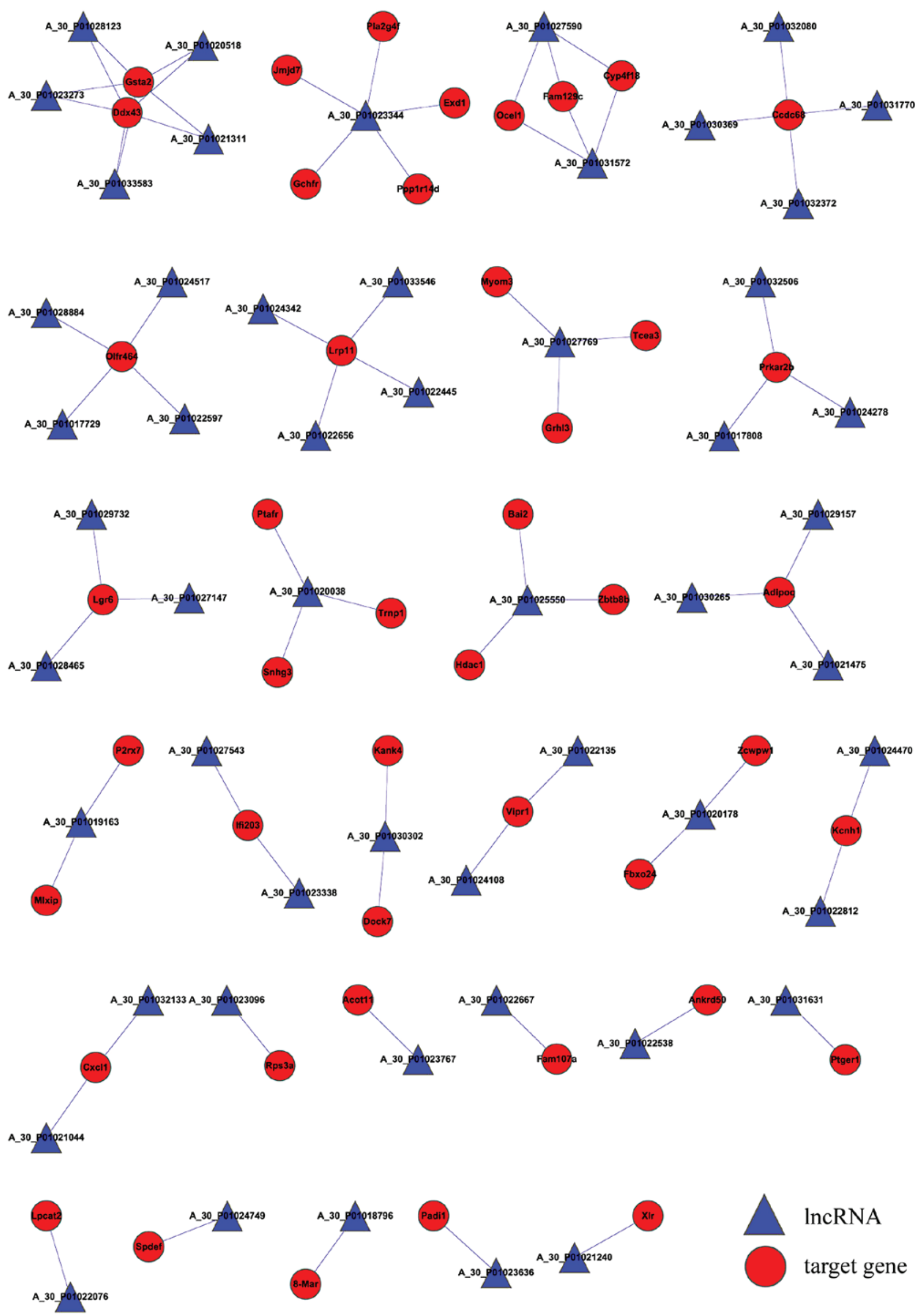

Figure 2. IncRNA-mRNA regulatory network. Circles represent genes and triangles represent lncRNAs; lines represent the association between the two types of regulation (solid line represents a cis-regulation, the dashed line represents the trans-regulation). IncRNA, long non-coding RNA. 
A

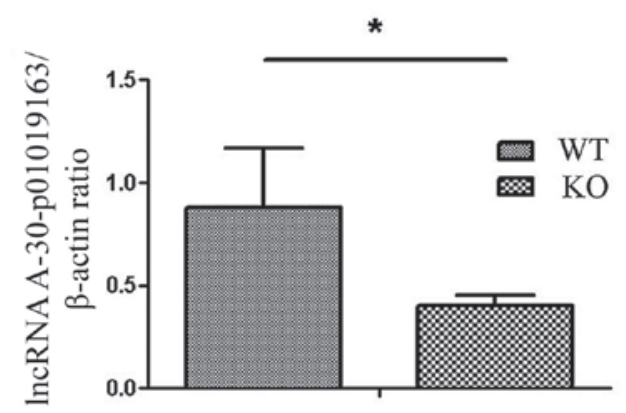

B

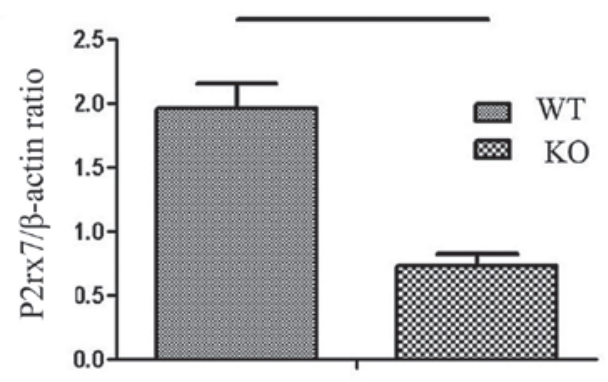

Figure 3. Validation of differentially expressed lncRNAs and mRNAs in ovary tissues from WT and $C R Y B B 2$ knockout mice. (A) RT-qPCR indicated lncRNA A-30-P01019163 was downregulated in ovary tissues from CRYBB2 KO mice; (B) RT-qPCR indicated P2rx7 was downregulated in ovary tissues from the $C R Y B B 2 \mathrm{KO}$ mice. ${ }^{*} \mathrm{P}<0.05$. RT-qPCR, reverse transcription-quantitative polymerase chain reaction; $\mathrm{KO}$, knock out; WT, wild-type.

using large scale cDNA library sequencing and next generation sequencing demonstrated abundant lncRNAs in mammals, however, not all lncRNAs are functional and only a relatively small proportion have been demonstrated to be biologically relevant $(11,23)$. For example, as of mid-2014, 197 lncRNAs have been functionally annotated in IncRNA databases (24). However, other lncRNAs may be translated into proteins (25). In addition, IncRNAs, unlike miRNAs, can down- or upregulate gene expression by targeting transcriptional activators or repressors (10), in addition to post-transcriptional regulation of protein expression.

In the current study differentially expressed lncRNAs in ovary tissues of $C R Y B B 2$ knockout mice were profiled and a total of 157 differentially expressed lncRNAs were observed. Furthermore, differentially expressed mRNAs were profiled and a total of 1,085 differentially expressed mRNAs in ovary tissues of $C R Y B B 2$ knockout mice were observed. GO and KEGG pathway analyses were performed to determine associations between these IncRNAs and mRNAs, and a number of these were subsequently verified using RT-qPCR. It was observed that lncRNA A-30-P01019163 and P2rx7 were significantly differentially expressed between 10 cases of ovary tissues from WT and CRYBB2 knockout mice. Thus, $C R Y B B 2$ knockout could downregulate expression of 1ncRNA A-30-P01019163 and, subsequently, suppress expression of the downstream gene P2rx7 and affect ovarian cell signal transduction, cell cycle, and ultimately ovarian development.
The current study is a proof-of-principle study and there are certain limitations. For example, it was not confirmed how 1ncRNA A-30-P01019163 regulates P2rx7 expression and the mechanistic investigation into how lncRNA A-30-P01019163-regulated P2rx7 expression mediates the effects of $C R Y B B 2$ on ovary development could be expanded on.

In conclusion, CRYBB2 regulates expression of different lncRNAs to influence ovary development. lncRNA A-30-P01019163 may affect ovarian cell cycle and proliferation by regulating P2rx7 expression in the ovary.

\section{Acknowledgements}

The authors would like to thank Shanghai Sensichip Infotech Co., Ltd. (Shanghai, China) for assistance with bioinformatic analysis and Medjaden Bioscience Ltd. for assisting in the preparation of the manuscript. The present study was supported in part by grants from the National Natural Science Foundation of China (grant nos. 81170834 and 81300748).

\section{References}

1. Zhang J, Li J, Huang C, Xue L, Peng Y, Fu Q, Gao L, Zhang J and Li W: Targeted knockout of the mouse betaB2-crystallin gene (Crybb2) induces age-related cataract. Invest Ophthalmol Vis Sci 49: 5476-5483, 2008.

2. Ganguly K, Favor J, Neuhüuser-Klaus A, Sandulache R, Puk O, Beckers J, Horsch M, Schädler S, Vogt Weisenhorn D, Wurst W and Graw J: Novel allele of crybb2 in the mouse and its expression in the brain. Invest Ophthalmol Vis Sci 49: 1533-1541, 2008.

3. Liedtke T, Schwamborn JC, Schröer U and Thanos S: Elongation of axons during regeneration involves retinal crystallin beta $\mathrm{b} 2$ (crybb2). Mol Cell Proteomics 6: 895-907, 2007.

4. Magabo KS, Horwitz J, Piatigorsky J and Kantorow M: Expression of betaB(2)-crystallin mRNA and protein in retina, brain, and testis. Invest Ophthalmol Vis Sci 41: 3056-3060, 2000.

5. Duprey KM, Robinson KM, Wang Y, Taube JR and Duncan MK: Subfertility in mice harboring a mutation in betaB2-crystallin. Mol Vis 13: 366-373, 2007.

6. Gao Q, Sun LL, Xiang FF, Gao L, Jia Y, Zhang JR, Tao HB, Zhang JJ and Li WJ: Crybb2 deficiency impairs fertility in female mice. Biochem Biophys Res Commun 453: 37-42, 2014.

7. Gao Q, Yang XY, Gu ML, Zhu RR, Zhang JJ and Li WJ: Effect of beta-B2 crystallin on ovary development and estrous cycle of mouse. Reprod Contracept 33: 217-223, 2013.

8. Hangauer MJ, Vaughn IW and McManus MT: Pervasive transcription of the human genome produces thousands of previously unidentified long intergenic noncoding RNAs. PLoS Genet 9: e1003569, 2013.

9. Faghihi MA, Modarresi F, Khalil AM, Wood DE, Sahagan BG, Morgan TE, Finch CE, St Laurent G III, Kenny PJ and Wahlestedt C: Expression of a noncoding RNA is elevated in Alzheimer's disease and drives rapid feed-forward regulation of beta-secretase. Nat Med 14: 723-730, 2008.

10. Goodrich JA and Kugel JF: Non-coding-RNA regulators of RNA polymerase II transcription. Nat Rev Mol Cell Biol 7: 612-616, 2006.

11. Mercer TR, Dinger ME and Mattick JS: Long non-coding RNAs: Insights into functions. Nat Rev Genet 10: 155-159, 2009.

12. Reinius B, Shi C, Hengshuo L, Sandhu KS, Radomska KJ, Rosen GD, Lu L, Kullander K, Williams RW and Jazin E: Female-biased expression of long non-coding RNAs in domains that escape X-inactivation in mouse. BMC Genomics 11: 614, 2010.

13. Yang Z, Zhou L, Wu LM, Lai MC, Xie HY, Zhang F and Zheng SS: Overexpression of long non-coding RNA HOTAIR predicts tumor recurrence in hepatocellular carcinoma patients following liver transplantation. Ann Surg Oncol 18: 1243-1250, 2011. 
14. Kogo R, Shimamura T, Mimori K, Kawahara K, Imoto S, Sudo T, Tanaka F, Shibata K, Suzuki A, Komune S, et al: Long noncoding RNA HOTAIR regulates polycomb-dependent chromatin modification and is associated with poor prognosis in colorectal cancers. Cancer Res 71: 6320-6326, 2011.

15. Niinuma T, Suzuki H, Nojima M, Nosho K, Yamamoto H, Takamaru H, Yamamoto E, Maruyama R, Nobuoka T, Miyazaki Y, et al: Upregulation of miR-196a and HOTAIR drive malignant character in gastrointestinal stromal tumors. Cancer Res 72: 1126-1136, 2012.

16. Huang MD, Chen WM, Qi FZ, Xia R, Sun M, Xu TP, Yin L, Zhang EB, De W and Shu YQ: Long non-coding RNA ANRIL is upregulated in hepatocellular carcinoma and regulates cell apoptosis by epigenetic silencing of KLF2. J Hematol Oncol 8: 50, 2015.

17. Kiefer JC: Epigenetics in development. Dev Dyn 236: 1144-1156, 2007.

18. Lee JT: Gracefully ageing at 50, X-chromosome inactivation becomes a paradigm for RNA and chromatin control. Nat Rev Mol Cell Biol 12: 815-826, 2011.

19. Zhang J, Huang CG, Li WJ, Weng W and Wang JQ: Establishment of a $\beta$ B2 crystallin gene knockout mice model. Acad J Sec Mil Med Univ 27: 1246-1248, 2006.
20. Livak KJ and Schmittgen TD: Analysis of relative gene expression data using real-time quantitative PCR and the 2(-Delta Delta C(T)) method. Methods 25: 402-408, 2001.

21. Jobby MK and Sharma Y: Calcium-binding to lens betaB2and betaA3-crystallins suggests that all beta-crystallins are calcium-binding proteins. FEBS J 274: 4135-4147, 2007.

22. Kang D, Hur CG, Park JY, Han J and Hong SG: Acetylcholine increases $\mathrm{Ca} 2+$ influx by activation of CaMKII in mouse oocytes. Biochem Biophys Res Commun 360: 476-482, 2007.

23. Dinger ME, Amaral PP, Mercer TR and Mattick JS: Pervasive transcription of the eukaryotic genome: Functional indices and conceptual implications. Brief Funct Genomic Proteomic 8: 407-423, 2009.

24. Amaral PP, Clark MB, Gascoigne DK, Dinger ME and Mattick JS: lncR NAdb: A reference database for long noncoding RNAs. Nucleic Acids Res 39 (Database Issue): D146-D151, 2011.

25. Smith JE, Alvarez-Dominguez JR, Kline N, Huynh NJ, Geisler S, $\mathrm{Hu} \mathrm{W}$, Coller $\mathrm{J}$ and Baker KE: Translation of small open reading frames within unannotated RNA transcripts in Saccharomyces cerevisiae. Cell Rep 7: 1858-1866, 2014. 